\title{
La ética de la responsabilidad de investigar para la salud en un país de ingresos medianos bajos: El Salvador
}

Virginia Rodríguez Funes, FACS

La medicina es la ciencia de las probabilidades y el arte de manejar las incertidumbres

Salvador Pita Fernández

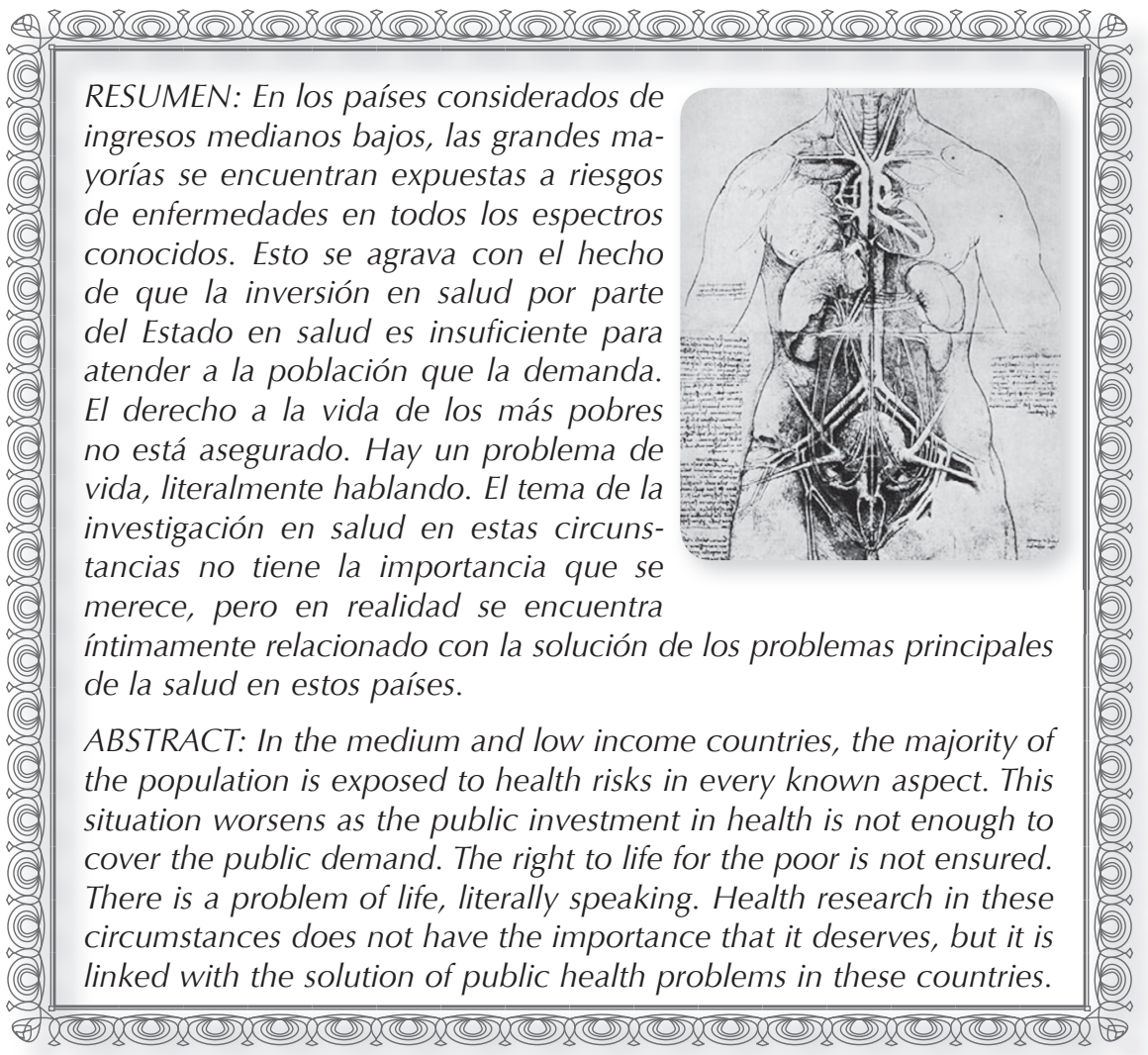




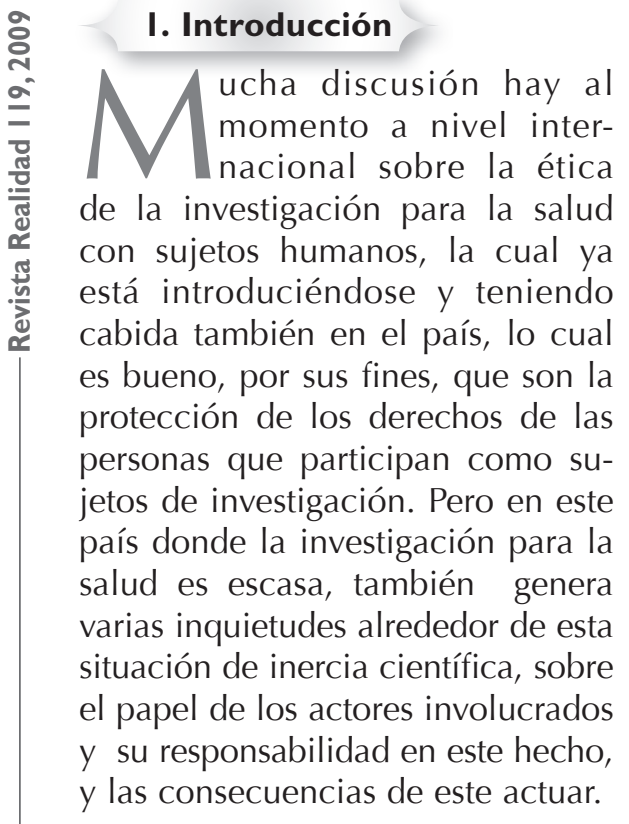

La investigación para la salud en El Salvador no ha retomado la importancia que debería tener como un medio de mejorar la salud de la población, a pesar de ser contada entre las herramientas necesarias para la planificación adecuada de estrategias de impacto, para la mejora de los niveles de salud de

\section{La medicina actual}

E n algún punto en el tiempo dentro de los últimos dos siglos previos al que nos encontramos, el mundo entero llegó al consenso de abordar la salud desde el enfoque científico, conocido como la "medicina occidental". Este consenso pudo haber ocurrido como uno de los tanto efectos de la transculturización durante la coloni- la población y para el abordaje directo de las patologías, preventiva o curativa.

$Y$ eso que El Salvador, siendo un país pequeño, pero que cuenta con siete facultades de Medicina, con tres sistemas proveedores de salud que sirven de plataforma para la formación de recurso médico especializado y dos instituciones que imparten maestrías de salud pública, se esperaría encontrar otra actitud con respecto al tema.

A nivel internacional, El Salvador se encuentra ubicado como uno de los países que no cuenta con marcos regulatorios ni con Políticas de investigación para la salud, de ciencia y tecnología que involucre la salud, ni de medicamentos, además de estar en la lista de los países que menos invierte en investigación desde su PIB y desde su concurso con patrocinantes externos.

Lo que nos ha llevado a una reflexión sobre la ética de la responsabilidad pública e individual sobre este fenómeno nacional.

zación europea al resto del mundo, también como efecto consecuente al asumir su responsabilidad por el manejo de enfermedades virales y bacterianas que afectaban a los colonizadores europeos y que eran diseminadas a las distintas poblaciones durante su paso por el resto de los continentes, empoderados aún más por el descubrimiento de la 
etiología de varias de ellas como el cólera, la tifoidea, el tétano, la neumonía y otras ${ }^{1}$. Aún civilizaciones milenarias que tenían sistemas terapéuticos avanzados y que actualmente siguen ocupando espacios alternativos de curación, como la acupuntura y la homeopatía, adoptaron el sistema médico occidental para tratar sus enfermedades.

Actualmente, con mucha dificultad podríamos concebir una sociedad categorizada como civili- zada sin que cuente, por lo menos, si no con un hospital, con una clínica donde haya una enfermera y un médico con un estetoscopio y medicamentos. Los indicadores con los que se mide el desarrollo de los pueblos incluyen el impacto de este tipo de atención en la población. Y el acceso de la población a este tipo de "medicina" o tratamiento está contemplado en el concepto del derecho a la salud, como un derecho humano de segunda generación.

\section{La medicina, como ciencia, evoluciona}

E $s$ igual de interesante que en el proceso de adopción de la medicina científica también se trasmitió la sensación que dicha "medicina" tenía respuesta para todos los problemas que afectan la salud de las distintas poblaciones e individuos, y que, si aún estas respuestas no existen, siempre habrá alguien quien partiendo del conocimiento del cuerpo humano, de cómo éste funciona y cómo funcionan los procesos de enfermedad, en poco tiempo las encontraría, pensamiento que ha sido fuertemente refutado por enfermedades como

el cáncer y la pandemia del Virus de Inmunodeficiencia Humana.

La medicina científica trajo consigo también la sensación de la unifactorialidad en la aparición de una enfermedad, aislada del contexto donde se encuentra el individuo, donde un tratamiento sencillo para la misma, como por ejemplo, que para la enfermedad $X$ existe la pastilla $X$ o la operación era suficiente, y además que estos manejos están bajo el dominio de los médicos, y muchos de éstos médicos como muchos profesionales de la salud, también lo consideraron así.

\section{Brechas del conocimiento}

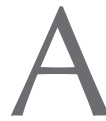
rchibald Cochrane, epidemiólogo inglés, en su libro Efectividad y eficiencia, que publicara en 1971, hizo un análisis de lo limitado y poco científicamente respaldado que era el conocimiento médico en ese momento, en cuanto a la terapéutica, y hacía un bosquejo histórico de cómo había impactado más las intervenciones en las determinantes socio-ambientales que la misma 
terapéutica sobre la epidemiología de un grupo poblacional dado. Pero igualmente señalaba que había una especie de simbiosis entre el paciente y el médico que se podía expresar de la siguiente manera: "El deseo de ayudar y el deseo de ser ayudado". El problema se agravó cuando se empezó a notar que esta posesión completa del conocimiento por los profesionales de la salud no era cierta, y entonces el paciente empezó a demandar más y más, haciendo que el médico utilizara medicamentos de los cuales todavía no se conocía su eficacia ${ }^{2}$ con tal de satisfacer la demanda.

Desde la publicación del libro de Cochrane, en los países de altos ingresos se aumentaron los esfuerzos para que la medicina use terapéuticas basadas en la evidencia.

La investigación para la salud también tuvo un auge en los últimos cincuenta años, motivada por esta necesidad de obtener más conocimiento, y de encontrar fundamento científico para las terapéuticas. Paralelamente, en la academia médica se generó la exigencia a la sociedad médica de producir investigación, como un mecanismo de desarrollo y superación profesional, el cual se reflejó en el dicho que se diseminó en los años 70 de "publish or perish" (Publica o perece). Lastimosamente esta exigencia, en lugar de abonar solo para bien, promovió también la producción de investigaciones en forma masiva, sin respeto a la calidad metodológica y con el fin de satisfacer las necesidades de los médicos para sí y no de las necesidades de salud de la población. Este movimiento académico no llegó a la sociedad médica de países de medianos y bajos ingresos, por lo que ninguno de los dos impactos tampoco llegó, ni el bueno ni el tergiversado.

Con el aumento del volumen de investigaciones para la salud que involucraban seres humanos, se intensificaron las denuncias de abusos hacia algunos sujetos de investigación por parte de los investigadores, y además empezó a inquietar el aparecimiento de tantos productos de avance científico y tecnológico. Esto provocó que, de forma reactiva, se fueran fortaleciendo los sistemas regulatorios alrededor de la investigación y que la bioética surgiera como una propuesta de "puente" entre las ciencias biológicas y médicas y las ciencias humanísticas. Fenómeno que tampoco vino en su tiempo a los países de medianos y bajos ingresos.

\section{El impacto de la pobreza en los sistemas de salud en el desarrollo científico}

- I impacto de las determinantes sociales de salud (DSS) en la carga de salud de las poblacio- nes ha sido ampliamente estudiado, tras la búsqueda de explicaciones científicas que hagan la conexión 
directa entre estas y la aparición de enfermedad, evolucionando hasta las teorías más recientes del "curso de la vida" ${ }^{3}$ y del trauma histórico ${ }^{4}$. Esta última noción mencionada se refiere al hecho de que aquellos pueblos que han sufrido circunstancias masivas y prolongadas de violencia colectiva presentan luego un importante aumento de enfermedades. Se trata de un marco macro para comparar la situación de poblaciones que tienen un peso aumentado de enfermedad entre ellas con la de otras que no han pasado por circunstancias similares.

Los tres elementos básicos de la teoría del trauma histórico son la experiencia traumática histórica, la respuesta histórica al trauma y la transmisión intergeneracional del trauma histórico. Las poblaciones así afectadas presentan un importante aumento de la carga de enfermedad.

En El Salvador, clasificado por los organismos internacionales entre los países considerados de bajomedianos ingresos ${ }^{5}$, estas determinantes sociales de la salud (DSS) que inducen a la enfermedad no están controladas, manteniendo a las grandes mayorías de esta población en un estado de fragilidad y de alto riesgo. Estas personas se encuentran expuestas a estas DSS en todos los espectros conocidos: desnutrición, analfabetismo funcional, viviendas inadecuadas cuando las poseen, problemas de excretas, falta de agua potable, exposición a las inclemencias climatológicas, estrés, exposi- ción a malas condiciones laborales, viviendas en áreas contaminadas con metales pesados $u$ otros, violencia estructural, y violencia física y psicológica y al trauma histórico, entre otros, condicionando enfermedades que pueden ir desde un retardo en el crecimiento intrauterino de un bebé, embarazos de alto riesgo, diarreas, hasta cánceres, leucemias, víctimas de trauma, insuficiencia renal y otras.

$Y$ esto se agrava con el hecho que cuando esta población pierde su salud física tampoco cuenta con el beneficio de acceder a un sistema de salud que le ayude en la recuperación de su precaria salud previa, ya que la inversión en salud por parte del Estado es absoluta y relativamente poca $^{6}$, siendo insuficiente para atender a la población que la demanda. El derecho a la vida de los más pobres y de los menos pobres, pero siempre pobres, no está asegurado.

Hay un problema de aseguramiento de la vida, literalmente hablando, en este círculo vicioso del mantenimiento de la salud, pérdida de la salud y su recuperación.

O sea, que en la población pobre y de medianos ingresos podemos identificar problemas graves que atentan contra la vida: determinantes que promueven la enfermedad, no acceso a recuperar la salud, inequidad en la atención en salud, falta de políticas de medicamentos que aseguren su acceso. Pero estos temas han sido estudia- 
dos por intelectuales de la salud pública y no son el tema central de esta reflexión.

El tema de la investigación para la salud en estas circunstancias tan escandalosas es poco o nada visible, diría más bien hasta rechazado en muchas de estas reflexiones de la salud pública, siendo relegado por estas grandes preocupaciones como algo superfluo para la magnitud de los problemas imperantes, cuando la realidad es que se encuentra entre las herramientas necesarias para la solución adecuada y eficaz de estos problemas.

\section{Actitudes hacia la investigación en salud y su consecuencia}

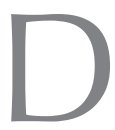
e lo que no se tomó conciencia en estos países pobres en el momento de aceptar el modelo científico para enfrentar la pérdida de la salud es que la ciencia es dinámica y evolutiva, y los conocimientos están en continuo movimiento de superación de los previos conceptos. Por lo que al adoptarla se debió adoptar también el compromiso de participar activamente en esta evolución y no quedarse esperando a que también las innovaciones sean importadas.

Por lo que una sociedad cuya medicina está basada en el modelo científico, su sociedad médica y paramédica académica y de atención directa tiene tres posibles actitudes, aunque de estas tres solo una se puede considerar ética, la que consiste en la conducción lógica, honesta y científica de la disciplina. Las tres actitudes que se pueden tomar, pero como mencionamos, que las dos primeras se contraponen con el deber ser, son: 1) Renunciar al pensamiento científico de la medicina, y aferrarse a los conocimientos obsoletos, que no evolucionan; 2) mantener el espíritu científico de la medicina, pero no invertir dinero ni tiempo para ser protagonistas de la búsqueda de respuestas y estar dependientes de que otros "países" lo hagan y consumir tecnología extranjera; 3) mantener el espíritu científico, invertir dinero y tiempo en crear un sistema nacional de investigación en salud (fortalecer la autoridad regulatoria, el marco ético de la investigación y la academia), y buscar respuestas a los problemas locales.

La primera actitud presenta los siguientes problemas morales:

En el ámbito de la ética de la responsabilidad personal': el médico al graduarse toma un compromiso que ha sido simbólicamente reflejado en el Ilamado juramento hipocrático, que recalca en el graduando su compromiso en el ejercicio de su profesión con pautas deontológicas hacia su relación médico-paciente, hacia la ciencia que ejerce, comprometiéndose a respetarla, respetar a sus profeso- 
res y trasmitir la profesión a todos aquellos que deseen aprenderla. En su relación con su paciente se compromete a darle la mejor calidad de atención posible, y recordando el concepto de calidad, dice: "es el grado en el cual los servicios de salud para individuos y poblaciones aumenta la similitud de la evolución en salud deseada y es consistente con el conocimiento actual de la profesión" ${ }^{\prime \prime}$, concepto de calidad que Ileva implícito el referente de atender al paciente con el estado actual del conocimiento. Con Io que al aferrarse a conceptos de manejo obsoletos se falta a este compromiso de atender con calidad.

Además, es una actitud muy cómoda de transformar la medicina en una profesión técnica automatizada de conceptos y prácticas que se hacen de forma repetitiva, sin fundamentos científicos que apoyen su quehacer, negándole a la sociedad el acceso a una respuesta adecuada a sus problemas de salud.

Otro de los problemas que se generan es que los espacios de incertidumbre existentes en el conocimiento se mantienen, lo cual impacta también en la negación al derecho a la salud de la población, al no intentar buscar de forma científica, respuesta a los problemas existentes. Puede que no se encuentren debido a nuestras limitaciones humanas, pero por lo menos no se demuestra un desinterés ante el dolor ajeno, sino un esfuerzo de buscar soluciones a sus problemas.
En el ámbito de la responsabilidad pública: el negar el papel primordial que juega la investigación dentro del quehacer de atención en salud y no tomar en cuenta la evidencia científica (los resultados de investigaciones de buena calidad) para la toma de decisiones, promueve la toma de decisiones arbitrarias, con la consecuente mal utilización de los escasos recursos con los que se cuenta en programas ineficaces y sin impacto.

Tomando en cuenta que el Estado es el que debe poner las reglas del juego en la atención en salud, éste debería preocuparse por asumir su papel como ente rector de la investigación para la salud, crear leyes, reglamentos y normas, que regulen esta actividad para el bien común, y que también cree políticas, que lleve a la designación de presupuesto que financien esta actividad y la creación de una institucionalidad que faciliten el quehacer investigativo en una sociedad, y proteja a esta misma de las Ilamadas investigaciones "safari" o de los investigadores inescrupulosos, haciendo que la investigación sea una práctica instalada y al servicio de la comunidad.

La segunda actitud, que es la adoptada en El Salvador y acepta el espíritu científico de la medicina, es la necesidad de la investigación como una herramienta de salud pública, pero, argumentando la poca capacidad económica para su desarrollo, se vuelve dependiente 
de que "otras sociedades" inviertan en investigación y les resuelva sus problemas, lo que en realidad refleja un desentendimiento del derecho a la salud por parte del Estado, de la Academia y de la sociedad proveedora de salud en general. Desglosando los problemas morales que esto produce, tenemos:

En el ámbito de la ética de la responsabilidad personal: Primero, el médico se compromete desde su graduación y cuando desee ejercer su profesión con la consecución de la más alta calidad de atención a su paciente. Dependiendo de su inclinación hacia la investigación, puede tomar dos posiciones: si no se encuentra inclinado como persona en la capacidad de dedicar parte o la totalidad de su tiempo para la búsqueda de nuevo conocimiento, por lo menos debe comprometerse con la búsqueda, lectura y utilización de los nuevos resultados de la investigación producida por otros cuando cuenten con buen nivel de evidencia científica.

El médico que sienta inclinación hacia dedicarle tiempo a la generación del nuevo conocimiento, debe primero conscientemente capacitarse formalmente en metodología de la investigación, conocer, aprender y aprehender la ética de la investigación, y respetar el mínimo regulatorio existente en el país. Igualmente debe comprometerse con las necesidades del país y buscar realizar estas investigaciones hacia la consecución de soluciones a los problemas prioritarios de salud pública que más afectan a las grandes mayorías.

Otro punto importante a mencionar como problema ético de la responsabilidad personal es cuando el médico se interesa en participar como investigador recolector de datos para una compañía extranjera, sea esta una universidad, una organización de investigación por contrato o una casa farmacéutica transnacional. Situación a la que se prestan algunos médicos con el fin de hacer investigación, lo cual en sí no es un problema ético, ya que el Estado le niega la posibilidad de desarrollarse en esa área. El problema ético se genera cuando el médico se presta a desarrollar la investigación no con el fin de traer un beneficio directo a la población, ya sea porque se está participando en un estudio de una terapéutica para una enfermedad que no es un problema de salud local o porque el medicamento probado al final no va a ser accesible para la población local; también cuando con tal de realizar la investigación no le preocupa violar los códigos internacionales de la ética de la investigación exponiendo a sus propios conciudadanos a riesgo, y defiende la posición de la compañía trasnacional como por ejemplo de que no cumpla con el "valor" de la continuidad del tratamiento; y también cuando no es honesto consigo mismo y se vuelve un "maquilador" de recolección de datos con el fin último de añadir su participación en dicha actividad en su hoja de vida. 
En el ámbito de la ética de la responsabilidad pública: A partir de la revolución industrial, y a medida que los países de altos ingresos se industrializaban más, las diferencias en la epidemiología entre estos países ricos y los países pobres fueron apareciendo y aumentando, tal como hemos visto, por un cambio de las DSS de las poblaciones. Los contextos en los que los investigadores en salud de los países ricos se mueven son distintos a los de nuestro país, sin tomar en cuenta además que tienen a su disposición alta inversión para investigación destinada a buscar respuestas a los problemas que asedian a sus países, o en otros casos con fines de crear tecnología comercializable. Se han ido tecnologizando cada vez más y más, Ilegando hasta investigaciones tan sofisticadas e innecesarias como crear robots ayudantes de cirujanos o ambientes de salas de operaciones que no tienen mucho valor en el progreso de la salud en sí, si se analiza el coste/beneficio.

Todo este ordenamiento de prioridades de los países ricos, entre sus propios problemas y la comercialización de sus productos, lleva como una de las tantas consecuencias al abandono de la búsqueda de medicamentos para enfermedades infecciosas que sólo afectan a los pobres, como las denominadas enfermedades desatendidas (la malaria, antiparasitarios u otros), fenómeno lógico consecuente de un pensamiento "utilitarista".
Este fenómeno acuñó el término de la brecha 90/10, cuando se publicó en el reporte de la Comisión de la Investigación en Salud para el Desarrollo, en 1990, que se había estimado que solo alrededor del $5 \%$ de los recursos para investigación en salud a nivel mundial eran utilizados para resolver problemas de los países en desarrollo, que constituían el 93\% de la carga mundial de "mortalidad prevenible" .

A esto se añade la ironía que, aún siendo El Salvador un país de ingresos medianos bajos, con poco presupuesto para salud, se ve en la obligación de "consumir" la tecnología y la farmacología, que avanza cada día de forma más acelerada en salud, no pudiendo ponerla al servicio de todos por su alto costo, promoviendo la inequidad en el acceso.

Esto empeora también ante la situación de la aparición de medicamentos huérfanos, que son aquellas moléculas farmacológicas que ya existen para curar ciertas enfermedades, pero que ya no son producidas por los laboratorios internacionales porque no son un "buen comercio", ya que serían consumidas solo por estos países "pobres" afectados.

¿Puede el Estado salvadoreño, ante estas circunstancias, confiar en que los países desarrollados encontrarán las soluciones a nuestros problemas locales? No, por lo que se ha mencionado previamente, $y$ esta posición más bien se aleja de 
asumir su responsabilidad como Estado de invertir ante la búsqueda de solución a sus problemas locales. Recordando que la ética de la responsabilidad pública se define como el papel y los deberes de los Estados democráticos frente no solamente a temas universales como la ciudadanía y los derechos humanos, pero también con relación al cumplimiento de la Constitución de la República de cada país.

En la tercera actitud, el Estado debería ser ético ante su responsabilidad pública de cuidar el derecho a la salud y asumir la investigación para la salud como uno de los caminos importantes para disminuir la carga de enfermedad de su población, así como también, a mediano plazo, disminuir el costo de inversión de esa carga de enfermedad, sobre todo porque cuenta con escasos recursos económicos.

Al asumir y creer en la investigación para la salud, el Estado invertiría en la creación del Sistema Nacional de Investigación en Salud (SNIS), que permitiría generar inves- tigación dirigida a la solución de los problemas de salud del país.

EI SNIS resolvería la ausencia de estructura para la investigación a través de la promoción de las leyes ausentes como la Ley de Investigación en salud, la Ley de Medicamentos, para que se puedan desarrollar políticas y destinar fondos para el desarrollo de la investigación; que el Ministerio de Salud Pública y Asistencia Social asuma el papel como ente rector que le corresponde y cree la Autoridad Regulatoria Central de la investigación para la salud; el fortalecimiento y la legitimización del Comité Nacional de Ética de Investigación Clínica como ente regulador de la ética de la investigación; el fortalecimiento del CONACYT en general y en el área de salud como uno de los puntos clave del SNIS; el fortalecimiento de la Academia en la formación de profesionales para la investigación para la salud; la creación del Instituto o Centro o Agencia de Medicamentos para que se regule todo el proceso de desarrollo de medicamentos y biológicos necesarios para las enfermedades locales.

\section{Papel de la academia dentro de la responsabilidad pública}

iA caso la Academia no tiene responsabilidad en este problema? Las universidades que imparten la enseñanza de la medicina no cuentan con la infraestructura adecuada que permita atraer a estudiantes de medicina cuando están cursando las áreas básicas hacia la investigación en este nivel de creación del conocimiento (investigación básica o fundamental). En las áreas clínicas ocurre algo similar cuando Ilegan los estudiantes a un ambiente ajeno a la investigación, porque en los hospitales tampoco se promueve la investigación, concluyendo su carrera sin haberse encontrado en 
ningún momento expuestos a ese estímulo de curiosidad en la ciencia, de creatividad y de pensamiento crítico.

Esto impacta grandemente en el cerebro del estudiante de las profesiones de la salud en la conceptualización de la disciplina en que se forma y posteriormente se desenvolverá. A esto se suma que en esta etapa importante del aprendizaje no se enseña a buscar, evaluar, analizar y criticar de forma constructiva los procesos de consecución de esos logros por parte de los científicos extranjeros, lo cual de forma indirecta llevaría a promover la reproducción de estos procesos y a hacer investigación local.

Otro punto que impactó en este pensamiento crítico fue la suspensión temporal de las tesis de graduación para el doctorado de Medicina por muchos años en la Universidad de El Salvador, medida que fue revertida hasta hace pocos años, habiendo casi dos décadas con promoción de doctores sin dicho requisito.

Al momento, todas las facultades de medicina desarrollan una tesis de graduación, para la que solo cuentan con un curso de elaboración de tesis, un manual de elaboración de tesis y sus respectivas asesorías para un año. Excepto una universidad, en ningún manual de tesis se encuentra algo relacionado con el respeto a los principios regulatorios ni éticos en el desarrollo de las investigaciones. La formación en investigación en general en las universidades salvadoreñas se limita al principio del método científico con el fin de realizar las tesis de graduación. Este ejercicio no logra instalar en el estudiante las bases para desarrollar investigación para la salud en un futuro, ni en generar la cultura de investigación en los profesionales de la salud.

Posteriormente, ya graduado, el profesional de la salud inserto de forma laboral en el sistema de salud, tanto pública como privada, tampoco encuentra las condiciones necesarias para dedicar parte de su tiempo al desarrollo de esta actividad. Esto crea otro problema que no es tan visible, pero que está subyacente, y que presenta un problema serio para las sociedades pobres y que debe destacarse: ser pobres económicamente y no invertir en investigar para la salud predispone a poco desarrollo científico, en este caso, en el ámbito de salud.

También hay otro problema ético no visualizado dentro de este contexto: el desarrollo de moléculas farmacológicas se ha tornado muy caro para las grandes compañías farmacéuticas transnacionales, por lo que desde hace dos décadas están visualizando a los países pobres como lugares donde pueden realizar las investigaciones de sus molécula a bajo costo, utilizando a los médicos como "maquiladores" de datos a través de la inclusión de pacientes. Se consideran como "maquiladores" ya que estos mé- 
dicos no cuestionan la metodología utilizada en estos protocolos o proyectos, se les paga mucho menos de lo que se les pagaría a los médicos en los países sedes de estas compañías,, se implantan con mayor fluidez en los países con falta de regulaciones o regulaciones flexibles, ya que no existen entes regulatorios, no tienen que adquirir compromisos con él durante y después de la investigación porque no hay entes regulatorios. Resumiendo: hay una permisividad para que estos países sin regulaciones o regulaciones flexibles sean fácilmente victimas del "imperialismo ético" y del "doble estándar moral" en la investigación con fármacos.

La falta de compromiso con el después es lo delicado también, ya que al finalizar los estudios de desarrollo de medicamentos, una vez comercializados éstos, tienen tan alto costo que no les permite ser accesibles para la población del país que ayudó a llevarlo al mercado. Contrario sería si, como país, se tomara la tercer actitud mencionada, la de compromiso con la medicina y con la investigación, se contara con la capacidad de investigar e innovar, creando nuestras propias moléculas farmacológicas y biológicos y patentándolas. No sólo servirían para atender a nuestra población enferma y producirlos a costos accesibles para los escasos recursos del país, sino también para ser solidarios con todos los países pobres ofreciéndoles medicamentos y biológicos a bajo costo y de alta calidad.

\section{Consecuencias de "no investigar en salud"}

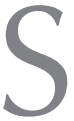
i bien la salud de las personas es responsabilidad de todos los ciudadanos del país, como lo menciona la Constitución salvadoreña, la responsabilidad de los médicos es dar atención con calidad, ser eficientes con los escasos recursos existentes destinados para salud, gestionar los recursos necesarios para solucionar los problemas de salud con quien corresponda, generar el conocimiento científico en salud.

Haber delegado la investigación para la salud a un plano fuera de las acciones prioritarias en salud, por cuales causas fuera, nos ha generado los siguientes problemas:

Primero: el desconocimiento de la verdadera epidemiología de nuestro país. Conocer por qué, de qué, y cómo evolucionan las enfermedades en la población salvadoreña para desarrollar programas de prevención, detección temprana y curación en esas enfermedades que más afectan. Su desconocimiento nos hace tener programas de salud inadecuados a las necesidades, aceptar las propuestas de intervención elaboradas por organismos internacionales sin cuestionar $y$ 
que, por lo tanto, no impactan en mejorar la salud, la calidad de vida y la sobrevida de los salvadoreños.

Segundo: desconocer los factores de riesgo causales de las enfermedades que nos afectan no permite la elaboración de programas de prevención, ni atacar de forma contundente las determinantes sociales y ambientales que las condicionan.

Tercero: al desconocer cómo sería la mejor manera de tratar, recuperar y rehabilitar a los que se enferman de nuestras patologías más frecuentes y en los estadios en que encontramos a nuestros enfermos, no se está ofreciendo a los ciudadanos salvadoreños la mejor calidad de atención posible, la cual está íntimamente ligada a satisfacer las expectativas del paciente y hacerlo con el conocimiento actualizado.

El desconocimiento de la eficacia y seguridad de los medicamentos nos hace caer como médicos en dos grandes fenómenos: primero, al dar medicamentos de los que se desconoce su eficacia y seguridad, se expone a los pacientes a efectos más riesgosos que beneficiosos y no se puede hacer la evaluación de riesgo/beneficio que se debe hacer previamente para ofrecer terapéuticas a los pacientes para sus tomas de decisiones; segundo, al perpetuar el vacío de un conocimiento que sería necesario para aliviar la salud en nuestro contexto, se abandona a los pacientes a que sufran de esas patologías sin ninguna atención en salud, negándoles el derecho a la salud.

El desconocimiento de la responsabilidad que tiene la sociedad médica de investigar en salud, que no le fue fomentada durante su formación académica, se encuentra de forma paralela con la actitud de seguir ejerciendo la medicina "como se ha hecho siempre aquí", impactando de forma negativa en la población salvadoreña. Desconocimiento que ha tenido un origen multifactorial, pero del que se debe despertar en algún momento y hoy es siempre el momento adecuado.

No investigar en salud, por lo tanto, nos crea muchos problemas morales que atentan contra el derecho a la salud de la población salvadoreña: persistencia de la inequidad en el acceso, dificultad al acceso a los medicamentos, programas que no solucionan las DSS, haciendo que los logros en salud no sean perdurables, etc., tomando en consideración que el derecho a la salud es el reflejo de uno de los principales valores morales del paradigma bioético en el contexto de la salud pública de cada país ${ }^{10}$, donde hay que aprender y poner en práctica valores y es cuestión obligatoria saber cómo cumplirlo 


\section{Recomendaciones}

L

a pasividad relacionada con la investigación ha dejado al país sumergido en una sociedad acrítica, no analítica y sin cultura de investigación en el área de la salud, que cubre las siguientes áreas:

1. Desconocimiento, indiferencia e irrespeto ante los principios metodológicos, éticos y legales de la investigación para la salud con sujetos humanos.

2. Desconocimiento del mecanismo y el proceso de desarrollo de moléculas farmacéuticas.

3. Falta de un ente regulador nacional de las investigaciones en salud.

4. Falta de un sistema nacional de investigación en salud y de política nacional de investigación en salud con la consiguiente falta de presupuesto para incentivar la investigación.

5. Realización de investigaciones para cumplir con requisitos de graduación o deseo de ganar un premio, pero no para llenar un espacio o vacío del conocimiento.

6. Falta de fomento del pensamiento crítico, analítico y creatividad en el formando para las disciplinas de la salud.

Mencionando al padre Ignacio Ellacuría, cuando afirma que la inteligencia posee la estructura formal de "aprehender la realidad y enfrentarse a ella", que se puede desdoblar en tres dimensiones: Una, intelectiva: hacerse cargo de la realidad; segunda, una dimensión ética: cargar con la realidad, y, por último, una dimensión práxica: encargarse de la realidad ${ }^{11}$; y haciéndose cargo de esa realidad de nuestro país, al tener el conocimiento de la enfermedad de la población más desprotegida en su integralidad, frecuencia, causa, prevención, curación y rehabilitación por datos fidedignos, podríamos pasar a la dimensión ética de cargar con la realidad para hacer la praxis de encargarse de ella a través de la construcción de programas certeros para combatir las mayores causas de muerte de los ciudadanos y mejorar su calidad de vida combatiendo de raíz las determinantes sociales de salud, dentro de nuestras capacidades económicas: desarrollando nuestras propias moléculas científicamente elaboradas y con calidad y permitir a su vez el desarrollo humano, en este caso científico, de la comunidad médica de el país.

Por lo que podemos finalizar diciendo que la promoción de la investigación para la salud como una política de mejora de la salud de un país, sobre una plataforma regulatoria y ética y académicamente estructurada para la consecución del "bien común", impactará en la calidad de salud de la población, disminuyendo la inequidad, mejorando la calidad de atención, 
mejorando el acceso a la salud y, como fin último, cumpliendo con el derecho a la salud en su concepto amplio. Como consecuencia, prote- gerá el derecho a la vida, por lo que ya es tiempo de que todos tomemos nuestra parte de responsabilidad y actuemos.

\section{NOTAS}

1 http://www.historylearningsite. co.uk/history_microbes.htm

2 Cochrane AL. Efectividad y eficiencia. Reflexiones al azar sobre los servicios sanitarios. Asociación Colaboración Cochrane Española, 2000.

3 Justo L. Bioética y salud en América Latina. http://www.redbioetica-edu. com.ar

4 Sotero M. A conceptual model of historical trauma: implications for Public Health Practice and Research. Journal of Health Disparities Research and practice 2006; 1(1):93108

5 http://web.worldbank.org/WBSITE/ EXTERNAL/DATASTATISTICS/ 0,,contentMDK:20421402 pagePK: 64133150 piPK:64133175 theSite PK:239419,00.html
6 Política Nacional de Salud de El Salvador. Diario oficial septiembre, 2008.

7 Jonas H. Il principio responsabilità. Einaudi, Turín 1990.

8 Brooke R. Quality of Healt Care, Part 2. Measuring quality of care. BMJ 1996 Sep; 335 (13): 966-70

9 http://www.globalforumhealth.org/ Site/003 The $\% 2010 \% 2090 \% 20$ gap/001_Now.php

10 Garrafa V. Bioética y Salud Pública. http://www.redbioetica-edu.com.ar

11 Sobrino J. Fuera de los pobres no hay salvación. UCA Editores, 2008. 